\title{
The effect of interface movement and viscosity variation on the stability of a diffusive interface between aqueous and gaseous $\mathrm{CO}_{2}$
}

\author{
Bernard Meulenbroek, ${ }^{1}$ Rouhollah Farajzadeh, ${ }^{2,3}$ and Hans Bruining ${ }^{3}$ \\ ${ }^{1}$ Delft University of Technology, Delft Institute of Applied Mathematics, Mekelweg 4, \\ 2628 CD, Delft, The Netherlands \\ ${ }^{2}$ Shell Global Solutions International, 2288 GS, Rijswijk, The Netherlands \\ ${ }^{3}$ Delft University of Technology, Department of Geotechnology, Stevinweg 1, \\ 2628 CN, Delft, The Netherlands
}

(Received 20 July 2012; accepted 13 June 2013; published online 17 July 2013)

Carbon dioxide injected in an aquifer rises quickly to the top of the reservoir and forms a gas cap from where it diffuses into the underlying water layer. Transfer of the $\mathrm{CO}_{2}$ to the aqueous phase below is enhanced due to the high density of the carbon dioxide containing aqueous phase. This paper investigates the behavior of the diffusive interface in an enclosed space in which initially the upper part is filled with pure carbon dioxide and the lower part with liquid. Our analysis differs from a conventional analysis as we take the movement of the diffusive interface due to mass transfer and the composition dependent viscosity in the aqueous phase into account. The same formalism can also be used to describe the situation when an oil layer is underlying the gas cap. Therefore we prefer to call the lower phase the liquid phase. In this paper we include these two effects into the stability analysis of a diffusive interface between $\mathrm{CO}_{2}$ and a liquid in the gravity field. We identify the relevant bifurcation parameter as $q=\epsilon R a$, where $\epsilon$ is the width of the interface. This implies the (well known) scaling of the critical time $\sim R a^{-2}$ and wavelength $\sim R a^{-1}$ (The critical time $t_{c}$ and critical wavelength $k_{c}$ are defined as follows: $\sigma(k) \leq 0 \forall t \leq t_{c}$; equality only holds for $t=t_{c}$ and $k=k_{c}$ ). Inclusion of the interface upward movement leads to earlier destabilization of the system. Increasing viscosity for increasing $\mathrm{CO}_{2}$ concentration stabilizes the system. The theoretical results are compared to bulk flow visual experiments using the Schlieren technique to follow finger development in aquifer sequestration of $\mathrm{CO}_{2}$. In the appendix, we include a detailed derivation of the dispersion relation $\sigma(k)$ in the Hele-Shaw case [C. T. Tan and G. M. Homsy, Phys. Fluids 29, 3549-3556 (1986)] which is nowhere explicitly given. (C) 2013 AIP Publishing LLC. [http://dx.doi.org/10.1063/1.4813072]

\section{INTRODUCTION}

\section{A. Carbon dioxide sequestration}

Efficient storage of carbon dioxide $\left(\mathrm{CO}_{2}\right)$ in aquifers requires dissolution in the aqueous phase. Indeed the volume available for gaseous $\mathrm{CO}_{2}$ is less than for dissolved $\mathrm{CO}_{2}$ and the virtual density of dissolved $\mathrm{CO}_{2}$ is around $1330 \mathrm{~kg} / \mathrm{m}^{3}$ (Ref. 1) leading to more storage space than in the supercritical state $\left(<600 \mathrm{~kg} / \mathrm{m}^{3}\right)$.

When $\mathrm{CO}_{2}$ is injected into an aquifer the competition between viscous, capillary, and buoyancy forces determines the flow pattern. Due to buoyancy forces, $\mathrm{CO}_{2}$ migrates upwards and is trapped under the cap rock due to the capillary forces. ${ }^{2}$ An interface between a $\mathrm{CO}_{2}$-rich phase and brine exists. Then $\mathrm{CO}_{2}$ dissolves into brine by molecular diffusion. The transition zone between the water at the gas-water interface and water without dissolved $\mathrm{CO}_{2}$ is called the diffusive interface. Dissolution of $\mathrm{CO}_{2}$ in brine increases the density of the brine phase. ${ }^{1}$ When a brine column is overlain by $\mathrm{CO}_{2}$ in 


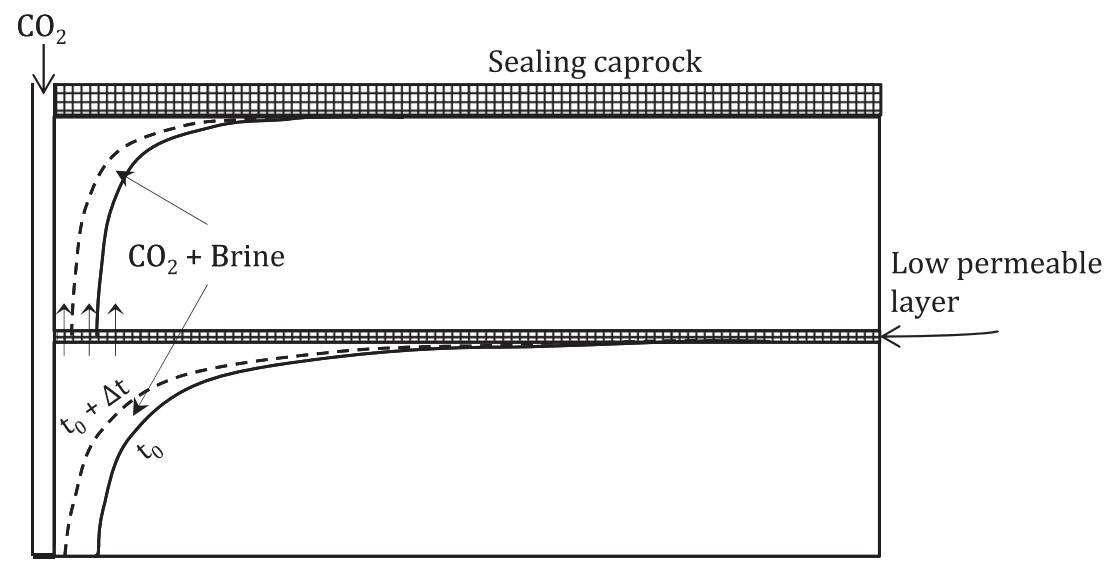

FIG. 1. Transfer and the role of interface movement.

a homogeneous aquifer ${ }^{3,4}$ the development of gravitationally unstable fingers greatly accelerates the dissolution rate thus making solubility trapping an important mechanism for long-term sequestration of $\mathrm{CO}_{2}$ in aquifers, ${ }^{5,6}$ see Fig. 1. The purpose of the paper is to understand mixing of $\mathrm{CO}_{2}$ with underlying brine for sequestration in aquifers or underlying oil for solvent based enhanced oil recovery. The underlying mechanism in each of these applications is that gaseous $\mathrm{CO}_{2}$ rises quickly to the top of the reservoir but due to capillary effects it gets trapped below low permeable layers. Initially the interface moves downward until the gas pressure builds up sufficiently to overcome the capillary forces and the $\mathrm{CO}_{2}$ bank moves upward and reaches the top layer. At the interface, mixing in the liquid region is influenced by natural convection effects that arise due to the fact that the liquid with $\mathrm{CO}_{2}$ dissolved in it has a higher density than the liquid without $\mathrm{CO}_{2}$. Consequently a mixing transfer occurs at the interface, which is moving initially downward and subsequently upward. The mixing rate is also influenced by the movement of the interface and the effect of $\mathrm{CO}_{2}$ on the viscosity. In convection dominated systems, the instability is determined by the relation $M>G+1$, where $M$ is the mobility ratio, $G$ is the gravity number. A system is stabilized in vertically upward movement by a higher viscosity and higher density displacing fluid. However in diffusion dominated systems, the stability, with and without interface movement, must be analyzed.

Several papers ${ }^{7-9}$ have described the conditions of instability of the $\mathrm{CO}_{2}$-water interface and onset of convective currents in porous media. Traditionally all models neglect viscosity variations and also the interface movement, while some theories (supported by experiments) suggest that interface cannot be assumed fixed. ${ }^{10,11}$ This paper extends the current numerical-linear-stability analyses by considering the interface movement. We also consider viscosity effects, because the dissolution of $\mathrm{CO}_{2}$ changes the viscosity of the liquid phase. There is an adverse relationship between the viscosity of an oleic phase and its $\mathrm{CO}_{2}$ content, while the viscosity of the aqueous phase increases when its $\mathrm{CO}_{2}$ content increases.

\section{B. Previous work on the stability of diffusive boundary layers}

The idea of a (conventional) stability analysis is as follows. A zeroth order state $\mathbf{u}^{0}(z)$, e.g., the concentration is found; this state is perturbed by $\mathbf{u}^{1}(x, y, z, t)$, where $\mathbf{u}^{1}$ represents, e.g., the noise. The idea is to decompose the perturbation in Fourier components $\mathbf{u}^{1}=\mathbf{f}(z) e^{i\left(k_{x} x+k_{y} y\right)+\sigma t}$ and to look for the fastest growing mode with growth rate $\sigma(k)$, since this mode will determine the stability of the system. In the case of a diffusive boundary layer however this method is not applicable, because the basic state depends on $t$. Depending on the specific boundary conditions, we have either

$$
c_{f}^{0} \sim \sin (n \pi z) e^{-n^{2} \pi^{2} t} \text { or } c_{i}^{0} \sim e^{-z^{2} /(4 t)},
$$

where $c_{f}^{0}$ is the solution of the diffusion equation in a finite domain, whereas $c_{i}^{0}$ is the solution in an infinite domain. This means that standard linear stability analysis is not applicable to this problem. 
A few methods have been used to address this problem, from which two of them will be discussed in more detail. The first method is the so called quasi steady state approximation (QSSA). ${ }^{12,13}$ The base state is considered "frozen in" at a certain time $t_{0}$ and the usual linear stability analysis is performed, where $t_{0}$ enters the calculation as a parameter. Such an approach is only valid if the perturbations grow on a faster timescale than the steady state.

Another approach is called amplification theory (AT). ${ }^{9,14,15}$ The idea is to expand the perturbed quantities in the streamwise direction in the eigenfunctions $c_{f}^{0}$, i.e.,

$$
\mathbf{u}^{1} \sim a_{n}(t) \sin (n \pi z) .
$$

Subsequently we multiply the resulting equations by $\sin (m \pi z)$ and integrate with respect to $z$ to obtain a coupled set of ordinary differential equations (ODE's) for $a_{n}$. Those ODE's are solved numerically. One has to be careful because this problem is not self-adjoint. ${ }^{14}$ It is not clear how to discriminate between stability and instability in this case. Usually the quantity

$$
\Theta(t)=\int c^{2}(t, z) d z / \int c^{2}(0, z) d z
$$

is defined to measure (in)stability. Some authors define $\Theta=1000$, others $\dot{\Theta}=0$ as the criterium for stability. This method will tend to overestimate critical times compared to the "normal" stability analysis, which already signals instability if only one mode is unstable. A more extensive overview of the different methods can be found in Emerging Topics in Heat and Mass Transfer in Porous Media by Rees et al. ${ }^{16}$

The diffusive instability is driven by a density gradient, which can be due to either temperature difference or a concentration gradient. We will not discriminate between those two here, since the resulting equations are basically the same. We will discuss two classes of problems. In Sec. I B 1, a few Hele-Shaw problems are discussed: a fluid with high density/viscosity invades a fluid with lower density/viscosity.

In Sec. I B 2 we will discuss our system. A boundary layer of finite width is destabilized due to gravitational (and viscous) effects. The width of the diffusive layer $W_{d i f}$ is much smaller than the height $H$ of the reservoir: destabilization takes place long before the bottom of the reservoir is reached: $W_{d i f} \ll H$ (or in dimensionless form $\epsilon=\frac{W_{d i f}}{H} \ll 1$ ). Physically this means that the width of the boundary layer is finite instead of infinite; mathematically this results in boundary conditions at $z=0$ instead of $z=-\infty$, which is the case for the Hele-Shaw problem. Although the systems may seem similar, analysis will reveal some crucial differences.

\section{Hele-Shaw problem}

In this problem a liquid with high concentration invades a liquid with low concentration with a certain velocity $U$. The stability of the diffusive boundary layer between the two states is studied using the QSSA. ${ }^{12,13,17}$ It is possible to find an analytical expression for the dispersion relation $\sigma(k)$ in the case of a sharp interface. This dispersion relation is given by several authors. ${ }^{12,13,17}$ Numerically the dispersion relation was studied in the latter papers for $t_{0}>0$. To the best of our knowledge a detailed derivation of this dispersion relation does not exist in the literature. We rederived the analytical expression for the dispersion relation, which can be found in Appendix B. It is not possible to find similar analytical expressions in our case. This is due to the fact that the boundary conditions are moved to zero instead of minus infinity.

Darcy's law has to be replaced by Stokes' equation whenever the assumption $\lambda \gg d$, where $d$ denotes the distance between the two parallel plates. In this case, Darcy's law is often replaced by the Brinkman equation: ${ }^{18}$

$$
\nabla p=\frac{-\mu}{k} \mathbf{v}+\mu^{\prime} \Delta \mathbf{v}
$$

which interpolates between Dacry's law and the Navier-Stokes equations without inertial terms, where the last term on the RHS of Eq. (2) is called the Brinkman correction. In general the Brinkman correction is a nonlocal operator of the gap-averaged velocity. ${ }^{19}$ In the long-wave limit, this correction 
can be simplified and effectively a factor of $\frac{6}{5}$ has to be added to the last term of Eq. (2). This model was analyzed in Ref. 20 and 21, where $\lambda \sim \frac{1}{R a}$ was found whenever $\lambda \gg d$ and $\lambda \sim d$ as $\lambda \ll d$. A good comparison to both numerical and experimental results was found.

In Ref. 22, the influence of the velocity $U$ on the diffusion coefficient was studied. An analytic expression for the dispersion relation was found in terms of a parameter $\eta$ which depends (among others) on $R$ (see Eq. (19)). Whenever $\eta$ passes a certain value, the dispersion relation exhibits a strong change (large wavenumber are no longer stable), which was verified experimentally in Ref. 23.

In Ref. 24, the linear stability predictions of the Navier-Stokes-Darcy (NSD)equation are compared to three dimensional lattice Bhatnagar-Gross-Krook (BGK) simulations. A characteristic length $L_{M R T}=\left(\frac{2 k_{0} H}{\varphi R a}\right)^{1 / 3}$ is introduced (the miscible Rayleigh Taylor length); the Darcy model for the Hele-Shaw cell is valid whenever the thickness of the cell $h \ll L$, the unbounded geometry case is obtained in the opposite limit $h \gg L$. The NSD-approach recovers both limits and also gives a good approximation in the intermediate range of cell thicknesses.

We have to be careful when we compare our results to the Hele-Shaw problem. The width of the boundary layer turns out to be the important parameter, which means that the aforementioned authors analyze a system, which is intrinsically physically different. One of the problems encountered is the absence of the separation of timescales, which is necessary for the justification of the approach. This means that one has to be careful when applying the QSSA to this particular problem.

Another interesting difference between the Hele-Shaw problem and the (semi)-infinite system is the behavior of the dispersion relation for small wavenumbers. In the Hele-Shaw case, the low wavenumbers are always unstable and the instability sets in for small $k$ (i.e., long wavelength). In the (semi-)infinite system however (see Sec. I B 2) and references therein) the instability sets in at some finite (nonzero) wavenumber $k_{c}$, which means that the destabilization takes place at a finite wavelength.

\section{Finite or semi-infinite systems}

In the case of a finite or semi-infinite system, a density gradient is created by either heating the system from below or by creating a larger concentration on top of the liquid. In both cases a small fluid layer with higher density is formed on top; the stability of this layer is studied. Elder ${ }^{25}$ observed that the scale of the flow is dominated by $\epsilon$, the width of the diffusive boundary layer. Looking at the scales of the equations $t_{c} \sim \epsilon^{2}$ and $\lambda_{c} \sim \epsilon$ were conjectured and $q=\epsilon R a$ as a critical parameter was observed. Rigorous analysis, however, "was left to the analysts." This analysis was performed by Foster $^{26}$ using the AT and varying the criterion described by Eq. (1). A band of critical wavelengths and the critical time were found as function of the Rayleigh number. This analysis was compared by Gresho and $\mathrm{Sani}^{27}$ to the QSSA. However both use the eigenfunctions $\sin (n \pi z)$, which are not suitable to describe $c_{f}^{0}$ if $\epsilon \ll 1$. Moreover Gresho and Sani ${ }^{27}$ use those eigenfunctions on top of the QSSA to compare both methods and find different results. It is incorrect to discard the QSSA on the basis of these findings. It is indeed true that the base state is changing fast, however, as we will show, the perturbations are changing even faster. The same AT method was used by Caltagirone ${ }^{15}$ with a different criterion for instability: the temperature instead of the velocity in Eq. (1). Scalings for critical time and wavenumber were found and stability was studied using the energy method. The same analysis is part of $\mathrm{Xu}$ et al. ${ }^{9}$ in the context of $\mathrm{CO}_{2}$ sequestration. They find the correct scaling relationships $t_{c}=C_{1} R a^{-2}$ and $\lambda_{c}=C_{2} R a^{-1}$. Solving the equations numerically, varying $R a$ and fitting their results they find $C_{1}=75$ and $C_{2}=96$.

The eigenfunctions for a finite system were replaced by the eigenfunctions of the diffusion operator in a semi-infinite domain in Riaz et al., ${ }^{7}$ which is preferable, since the domain is, after a proper rescaling, quasi semi-infinite. However only the first eigenmode is taken into account. Solving the resulting problem numerically for different Rayleigh numbers they observe $t_{c}=146 R^{-2}$ (notice the different rescaling of time in their paper) and $\lambda_{c}=90 \mathrm{Ra}^{-1}$.

A similar approach was taken by Ennis-King et al. ${ }^{8}$ who (apart from varying the permeability) also established $t_{c}=75 R a^{-2}$ and $\lambda_{c}=95 R a^{-1}$, which agrees with the results of Xu et al. ${ }^{9}$ 
A different approach was taken by Kim et al. ${ }^{28,29}$ who use propagation theory. Rescalings at the boundary layer are conjectured and the correct scalings of the critical wavelengths and times are found. This approach is close to the method used in this paper.

In this paper we are interested in the stability of the interface for $\epsilon \ll 1$, which means that our base state behaves like $c_{i}^{0}$. The base state $c_{i}^{0} \sim e^{-\eta^{2}}, \eta=\frac{z}{\sqrt{t}}$ and is truncated at $\eta=b$, where $b=10$ is chosen in the numerical examples. This means that the $\mathrm{CO}_{2}$ has advanced until $\eta=b$ (and not further), i.e., contributions $\sim e^{-100}$ are neglected. Since $\eta=\frac{z}{\sqrt{t}}$ this yields a relation between the width of the interface $\epsilon$ and the time needed to reach this width $\epsilon$,

$$
b=\frac{\epsilon}{\sqrt{t_{0}}} \Rightarrow t_{0}=\frac{\epsilon^{2}}{b^{2}} .
$$

This means that we can either fix the width of the interface $\epsilon$ or the time of interest $t_{0}$.

We will use the QSSA to study the stability of perturbations of the base state. Such an approach is only valid whenever there is a clear separation in timescales between the base state and the perturbed state. It is noted by many authors that the base state changes fast for small $\epsilon$ : Differentiating Eq. (3) yields $\frac{d \epsilon}{d t}=\frac{b^{2}}{2 \epsilon} \sim \frac{1}{\epsilon}$. We will show however that the convective instability sets in even faster: $\sigma \sim \frac{1}{\epsilon^{2}}$. This means that a clear separation of scales is achieved in this case, which justifies the QSSA. In principle this problem could be solved using a shooting method in the whole domain. This is problematic, since the base state $c_{i}^{0}$ changes fast in $(0, \epsilon)$ and vanishes if $z>\epsilon$. We prefer to split the domain into two regions: Region I (where $c^{0} \neq 0$ ) and Region II (where $c^{0}$ is effectively zero). We can solve the problem analytically in Region II, which leads to an effective boundary condition in Region I. We use a shooting method to solve the problem in Region I. The analysis identifies the bifurcation parameter as

$$
q=\epsilon R a \text {. }
$$

This is in fact the Rayleigh number with the width of the interface as a typical lengthscale, as observed by Elder. ${ }^{25}$ Then the previously observed scalings follow:

$$
t_{c} \sim R a^{-2}, k_{c} \sim R a .
$$

Notice that the (microscopic) parameter $q$ is a more logical bifurcation parameter than $R a$, because $R a$ is a macroscopic parameter. It uses the entire height of the system as the characteristic length whereas $q$ uses the width of the interface as the characteristic length. A similar technique is used in Refs. 30 and 31.

Remark: We use (partially) the same method as Kim, ${ }^{29}$ but they use a few conjectures, which follow naturally in our case.

The paper is organized as follows. In Sec. II the physical model is formulated and the dimensionless form of the governing equations is presented. The equations for the base state in the presence of a moving interface is solved, the linear problem in the QSSA is presented. The analysis in Region II is performed in Sec. III, which yields an effective boundary condition for Region I. This leads to a set of linear differential equations, which have to be solved numerically. The numerical results are given in Sec. IV and compared to the experimental results in Sec. V. We end the paper with concluding remarks.

\section{PHYSICAL MODEL}

\section{A. Formulation}

We consider a homogeneous and isotropic porous medium saturated with a fluid with a height $H$ and length $L$. The porosity of the porous medium is $\varphi$ and its permeability is $k_{0}$. Initially the fluid is at rest and there is no $\mathrm{CO}_{2}$ dissolved in the fluid. We assume no flow boundary at the sides of the porous medium. $\mathrm{CO}_{2}$ is continuously supplied from the top, i.e., the $\mathrm{CO}_{2}$ concentration at the top is kept constant. We disregard the presence of a capillary transition zone between the gas and the 


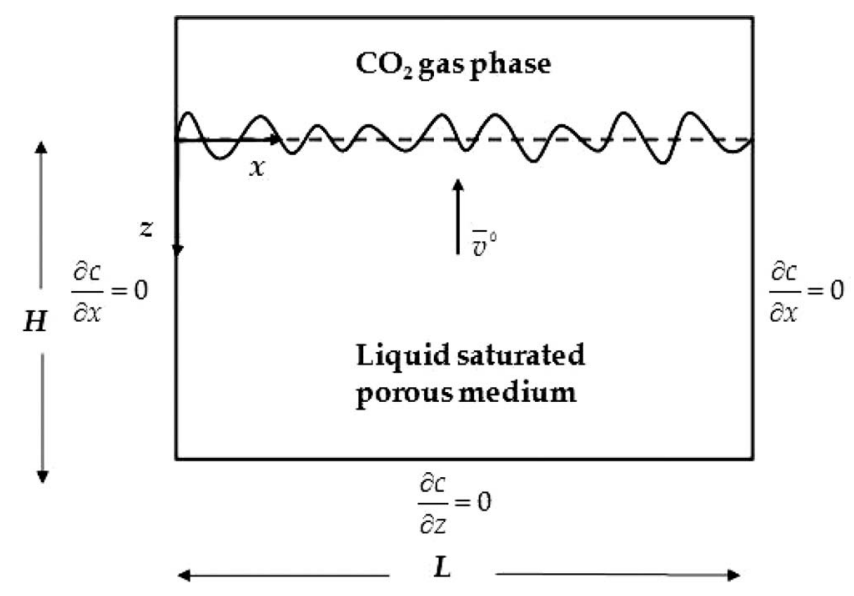

FIG. 2. Geometry of the system.

liquid phase. Hence we only model the liquid phase and the presence of the gas phase at the top is represented by a boundary condition for the liquid phase. We assume that $\mathrm{CO}_{2}$-liquid interface is sharp and the volume of the fluid changes due to mixing with $\mathrm{CO}_{2}$. The position of the top boundary is proportional to the width of the boundary layer, i.e., proportional to $\sqrt{t}: z_{t o p}=-A \sqrt{t}$ (the volume change is proportional to the change in the width of the boundary layer). The proportionality constant $A$ is estimated from the experiments ${ }^{32}$ and from typical field parameters. The fluid density, $\hat{\rho}$, and viscosity, $\hat{\mu}$, vary linearly with the $\mathrm{CO}_{2}$ concentration. The motion of the fluid is described by Darcy's law driven by a density gradient. Darcy's law is combined with the mass conservation laws for the components $\left(\mathrm{CO}_{2}\right.$ and either water or oil) to describe the diffusion and natural convection processes in the porous medium. We only expect a laminar regime since the Rayleigh number is small.

\section{B. Governing equations}

For the 2D porous medium depicted in Fig. 2 the governing equations can be written as

- Continuity equation

$$
\frac{\partial \hat{\rho}}{\partial \hat{t}}+\nabla \cdot(\hat{\rho} \hat{\mathbf{v}})=0,
$$

where $\hat{\mathbf{v}}=(\hat{u}, \hat{v}) ; \hat{u}$ and $\hat{v}$ are the velocities in the $x$ - and $z$-direction.

- Darcy's law

$$
\begin{gathered}
\hat{u}=-\frac{k_{0}}{\hat{\mu}} \frac{\partial \hat{p}}{\partial \hat{x}}, \\
\hat{v}=-\frac{k_{0}}{\hat{\mu}}\left(\frac{\partial \hat{p}}{\partial \hat{z}}-\hat{\rho} g\right),
\end{gathered}
$$

where $\hat{p}$ is pressure.

- Concentration

$$
\varphi \frac{\partial \hat{c}}{\partial \hat{t}}+\nabla \cdot(\hat{c} \hat{\mathbf{v}})=\varphi D\left(\frac{\partial^{2} \hat{c}}{\partial \hat{x}^{2}}+\frac{\partial \hat{c}}{\partial \hat{z}^{2}}\right),
$$

where $D$ is the molecular diffusion coefficient. 


\section{Dimensionless form of the equations}

We take $H$ as a characteristic length and define the dimensionless space and time as follows:

$$
\hat{x}=H x, \hat{z}=H z, \hat{t}=\frac{H^{2}}{D} t,
$$

which leads to a dimensionless velocity:

$$
\hat{u}=\frac{D}{H} u, \hat{v}=\frac{D}{H} v .
$$

It is however convenient to define a scaled velocity as follows

$$
\bar{u}=\frac{u}{\varphi}, \bar{v}=\frac{v}{\varphi}, \overline{\mathbf{v}}=(\bar{u}, \bar{v}) .
$$

Furthermore we set $\rho_{0}$ and $\mu_{0}$ as the density and viscosity of water, hence

$$
\hat{\rho}=\rho_{0} \rho, \hat{\mu}=\mu_{0} \mu, \hat{p}=p \rho_{0} g H .
$$

We rescale the concentration

$$
c=\frac{\hat{c}-c_{i}}{c_{0}-c_{i}}
$$

(where $c_{i}$ is the initial concentration and $c_{0}$ is the concentration at the interface).

Therefore Eqs. (4)-(7) become

$$
\begin{gathered}
c_{t}+\nabla \cdot(c \overline{\mathbf{v}})=c_{x x}+c_{z z}, \\
\bar{u}=-\frac{1}{\mu} p_{x} \overline{R a}, \\
\bar{v}=-\frac{1}{\mu}\left(p_{z}-\rho\right) \overline{R a}, \\
\frac{\partial \rho}{\partial t}+\nabla \cdot(\rho \overline{\mathbf{v}})=0,
\end{gathered}
$$

where

$$
\overline{R a}=\frac{k_{0} \rho_{0} g H}{\mu_{0} D \varphi} .
$$

Cross differentiate Eqs. (14) and (15) to eliminate pressure,

$$
(\mu \bar{v})_{x}-(\mu \bar{u})_{z}=\rho_{x} \overline{R a} .
$$

Both viscosity and density are assumed to depend linearly on $c$ hence

$$
\mu=1+R c, \rho=1+S c .
$$

The magnitude and sign of $R$ and $S$ depend on the type of the fluids. Generally positive $S$ implies that a light fluid with a low concentration is overlain by a heavier fluid, with a high concentration, which tends to destabilize the flow. However, positive $R$ means that a more viscous fluid overlays a less viscous fluid, which leads to more stability. Note that for $\mathrm{CO}_{2}$-water system both viscosity and density increase with increasing concentration and hence $R, S>0$. However for most $\mathrm{CO}_{2}$-oil systems the viscosity decreases with increasing concentration of $\mathrm{CO}_{2}$ and hence $R<0$ and $S>0$. The values for $\mathrm{CO}_{2}$-water system are estimated from Gmelin ${ }^{1}$ and Bando et al. ${ }^{33}$ as

$$
R=0.13, S=0.02,
$$

which means that $R$ is below the value for which velocity effects on the diffusion coefficient have to be taken into account. ${ }^{22}$

Notice that $\Delta \hat{\rho}=\hat{\rho}_{c=1}-\hat{\rho}_{c=0}=\rho_{0} S$, which implies

$$
\bar{R} a S=\frac{k_{0} \rho_{0} g H}{\mu_{0} D \varphi} S=\frac{k_{0} \Delta \hat{\rho} g H}{\mu_{0} D \varphi}=R a .
$$




\section{Boundary and initial conditions}

Initially we set

$$
\bar{u}=\bar{v}=c=0 \text {. }
$$

The boundary conditions are

$$
\bar{u}_{x}=c_{x}=0 \text { at } x=0, L / H
$$

and

$$
\bar{v}=0, c=1 \text { at } z=-A \sqrt{t} \text { and } \bar{u}=\bar{v}=0, c_{z}=0 \text { at } z=1 \text {. }
$$

\section{E. Base state solutions}

We are looking for zeroth order solutions of the form

$$
c(x, z, t)=c^{0}(z, t), \bar{u}=0, \bar{v}=v^{0}(z, t)
$$

with boundary conditions

$$
c^{0}(z, t)=1 \text {, at } z=-A \sqrt{t} \text { and } c_{z}^{0}(z, t)=0, v^{0}(z, t)=0 \text { at } z=1 .
$$

Notice that the dimensional form $\hat{v}^{0}$ can be retrieved from $\hat{v}^{0}=\frac{v^{0} D \varphi}{H}$.

Observe that Eq. (18) is automatically satisfied. Substitute ansatz (24) in Eq. (16) and use Eq. (19) to obtain

$$
S c_{t}^{0}+\partial_{z}\left(\left(1+S c^{0}\right) v^{0}\right)=0 \Rightarrow \partial_{z} v^{0}=-S\left(c_{t}^{0}+\partial_{z}\left(c^{0} v^{0}\right)\right) .
$$

Substitute ansatz (24) in Eq. (13) and use Eq. (19) to obtain

$$
c_{t}^{0}+\partial_{z}\left(c^{0} v^{0}\right)=c_{z z}^{0} .
$$

Combining Eqs. (26) and (27), we find

$$
v_{z}^{0}=-S c_{z z}^{0} \Rightarrow v^{0}=-S c_{z}^{0}
$$

since $v^{0}=c_{z}^{0}=0$ at $z=1$. Use Eq. (28) to eliminate $v^{0}$ from Eq. (27) to obtain an equation for $c^{0}$

$$
c_{t}^{0}+\partial_{z}\left(-S c^{0} c_{z}^{0}\right)=c_{z z}^{0}
$$

with boundary conditions $c^{0}(-A \sqrt{t})=1, c_{z}^{0}(1)=0$. Use the self similar coordinate $\eta=\frac{z}{\sqrt{t}}$ to derive an ODE for $c^{0}(\eta)$,

$$
-\frac{1}{2} \eta c_{\eta}^{0}+\frac{d}{d \eta}\left(-S c^{0} c_{\eta}^{0}\right)=c_{\eta \eta}^{0} \Rightarrow c_{\eta \eta}^{0}=\frac{-\eta c_{\eta}^{0}-S\left(c_{\eta}^{0}\right)^{2}}{1+S c^{0}}
$$

with boundary conditions $c^{0}=1, \eta=-A$, and $c_{\eta}^{0}=0, \eta=\frac{1}{\sqrt{t}} \approx \infty$ since we are interested in times of order $10^{-10}$. Physically this means that the interface is (infinitely) far away from the lower boundary. The ODE is solved numerically, see Figure 3.

\section{F. QSSA for the first order}

Perturb the base state

$$
\bar{u}=u^{1}(x, \tilde{z}, t), \quad \bar{v}=v^{0}(z, t)+v^{1}(x, z, t), c=c^{0}(z, t)+c^{1}(x, z, t),
$$

where $u^{1}, v^{1}$, and $c^{1}$ are small quantities. Inserting this in Eqs. (13), (16), and (18) yields up to first order

$$
S c_{t}^{1}+u_{x}^{1}+v_{z}^{1}+S c^{0} u_{x}^{1}+S c_{z}^{0} v^{1}+S c_{z}^{1} v^{0}+S c^{0} v_{z}^{1}+S c^{1} v_{z}^{0}=0
$$




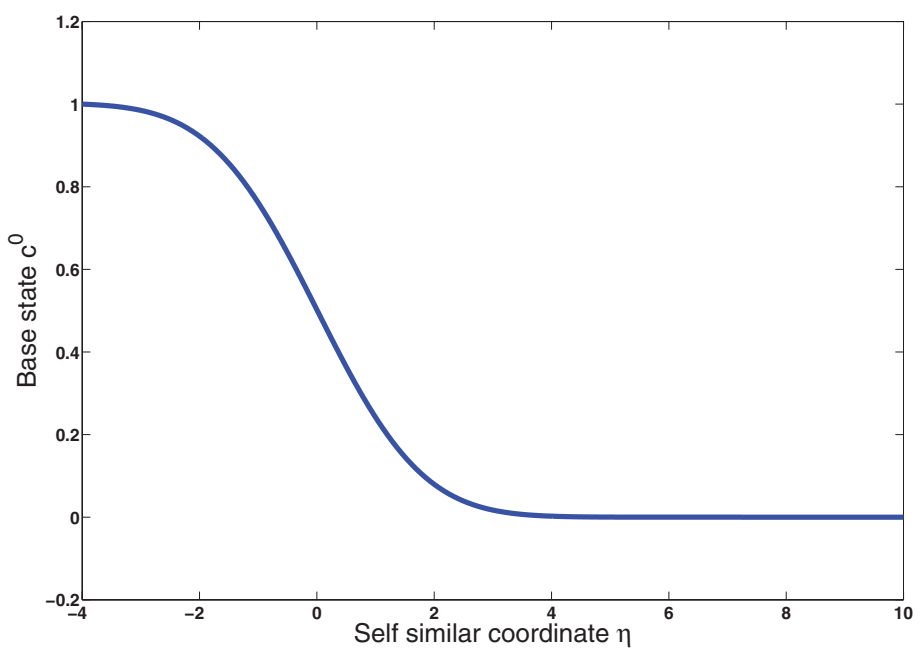

FIG. 3. The base state $c^{0}(\eta)$ for $A=4$, where $\eta=\frac{z}{\sqrt{t}}$.

$$
R c_{x}^{1} v^{0}+\left(1+R c^{0}\right) v_{x}^{1}-R c_{z}^{0} u^{1}-\left(1+R c^{0}\right) u_{z}^{1}=\overline{R a} S c_{x}^{1}=R a c_{x}^{1}
$$

and

$$
c_{t}^{1}+u_{x}^{1} c^{0}+v_{z}^{0} c^{1}+v_{z}^{1} c^{0}+v^{0} c_{z}^{1}+v^{1} c_{z}^{0}=c_{x x}^{1}+c_{z z}^{1} .
$$

We decompose the perturbed quantities in Fourier modes, i.e., $\sim e^{i k x}$. Furthermore we use the QSSA: the background state $c^{0}(z, t)$ is considered "frozen" on the timescale of the perturbations: $c^{0}\left(z, t_{0}\right)$. Perturbations $\sim e^{\sigma\left(t_{0}\right) t}$ are imposed and lead for every value of $t_{0}$ to a dispersion curve $\sigma\left(k ; t_{0}\right)$. We are interested in the onset of the instability, which means that we are looking for $t_{c}$ and $k_{c}$ such that $\sigma\left(k, t_{c}\right) \leq 0$ for all values of $k$. The critical wavenumber $k_{c}$ occurs at $\sigma\left(k_{c}, t_{c}\right)=0$. The QSSA only works as $\sigma>0$ (due to the time dependence of the underlying base state) which means that $k_{c}$ and $t_{c}$ are found using extrapolation of the data, see Sec. IV. The dependence of $\sigma$ on $t_{0}$ will be suppressed in the notation and we set

$$
u^{1}=\Pi(z, t) e^{i k x+\sigma t}, v^{1}=\phi(z, t) e^{i k x+\sigma t}, c^{1}=\psi(z, t) e^{i k x+\sigma t} .
$$

Substitution in Eq. (30) yields an expression for $\Pi(z, t)$,

$$
\Pi(z, t)=\frac{i}{k\left(1+S c^{0}\right)}\left(\phi_{z}+S\left(\sigma \psi+c_{z}^{0} \phi+c^{0} \phi_{z}+v_{z}^{0} \psi+v^{0} \psi_{z}\right)\right),
$$

which can be used to derive ODE's for $\phi_{z z}$ and $\psi_{z z}$

$$
\begin{gathered}
\psi_{z z}=\frac{\left(\sigma+k^{2}\left(1+S c^{0}\right)+v_{z}^{0}\right) \psi+v^{0} \psi_{z}+c_{z}^{0} \phi}{1+S c^{0}}, \\
\phi_{z z}=F\left(\phi, \phi_{z}, \psi, \psi_{z}\right) .
\end{gathered}
$$

An explicit expression for $F$ can be found in Appendix A.

\section{SPLITTING THE DOMAIN IN TWO PARTS}

In Eq. (3) we defined $b$

$$
t_{0}=\frac{\epsilon^{2}}{b^{2}}
$$


The constant $b$ is chosen such that the interface is located between $\tilde{z}=0$ and $\tilde{z}=\epsilon$. With this choice, the parameter $\epsilon$ is a measure of the width of the boundary layer as can be seen from Fig. 3, where $c^{0}$ is plotted as a function of $\eta=\frac{z}{t_{0}}$ at $t_{0}=b \epsilon^{2}, b=0.01, \eta=10 \frac{z}{\epsilon}$. Notice that the point $z=\epsilon$ corresponds to the point $\eta=10$ to the right in Fig. 3; the lower boundary, located at $z=1$ is much further away.

Since $\epsilon$ is small, $c^{0}$ is negligible in most of the domain (see Fig. 3), we see that we can be split the domain into two parts: $R_{1}=\{-0.1 A \epsilon \leq z \leq \epsilon\}$ and $R_{2}=\{z \mid \epsilon \leq z \leq 1\}$. Equations (33) and (34) can be simplified and solved analytically in $R_{2}$. Those analytical solutions yield two boundary conditions at $z=\epsilon$. The eigenvalue problem (33) and (34) is solved numerically in $R_{1}$. We have to solve two second order equations, which means that four initial conditions are required. Two initial conditions are given by Eq. (23) at $z=-0.1 A \epsilon$. A third initial condition can be chosen due to normalization (and is set to 1), the last one is the first shooting parameter. The eigenvalue $\sigma$ is the second shooting parameter. Equations (33) and (34) are integrated numerically up to $z=\epsilon$ where two conditions have to be met. Those two conditions determine both shooting parameters.

In Sec. III A, the analytical solutions in $R_{2}$ are derived, in Sec. III B, we solve the equations in $R_{1}$ numerically.

\section{A. Analytical solutions in $\boldsymbol{R}_{\mathbf{2}}$}

In $R_{2}$ we have $c^{0} \approx 0$ and Eqs. (33)-(34) reduce to

$$
\begin{gathered}
\left(\frac{d^{2}}{d z^{2}}-k^{2}\right) \phi=-\sigma S \psi_{z}-k^{2} \operatorname{Ra} \psi \\
\left(\frac{d^{2}}{d z^{2}}-l^{2}\right) \psi=0
\end{gathered}
$$

where $l=\sqrt{\sigma+k^{2}}$.

Solving Eq. (36) yields

$$
\psi=C_{1} e^{-l z}+C_{2} e^{l z}
$$

The integration constant $C_{2}$ is determined using

$$
\psi_{z}=0 \text { at } z=1 \Rightarrow C_{2}=C_{1} e^{-2 l} .
$$

In Sec. III B, we will show that $l \sim \frac{1}{\epsilon}$, which means that $C_{2} \sim e^{-2 / \epsilon}$. This can be neglected for small $\epsilon$. Therefore, we find

$$
\psi=C_{1} e^{-l z} \text { for } z>\epsilon
$$

Similarly we find for $\phi$

$$
\phi=D_{1} e^{-k z}+C_{1} \frac{l \sigma S-k^{2} R a}{l^{2}-k^{2}} e^{-l z} \text { for } z>\epsilon,
$$

where $D_{1}$ and $C_{1}$ are arbitrary constants. We have four matching conditions $\left(\phi, \phi^{\prime}, \psi, \psi^{\prime}\right)$ at $z=\epsilon$ : two are used to eliminate $C_{1}$ and $D_{1}$ and we are left with two conditions at $z=\epsilon$,

$$
\psi^{\prime}=-l \psi, \phi^{\prime}=-k \phi-\frac{l \sigma S-k^{2} R a}{l+k} \psi \text { at } z=\epsilon .
$$

\section{B. Analysis in $\boldsymbol{R}_{1}$ : A cascade of rescalings}

We have to solve Eqs. (33) and (34) with boundary conditions (39) and initial conditions (40) from Eq. (23)

$$
\phi=\psi=0 \text { at } z=-0.1 A \epsilon .
$$


Since $|z|<\epsilon(|A| \leq 4$ in the numerics) it is convenient to introduce a rescaling of the variables (see Sec. V). The mere rescaling of $z$, however, leads to a cascade of rescalings, which need to be done in order to have nontrivial solutions. This is due to the fact that the correct choice of the lengthscale in the diffuse regime is not $H$ but $\epsilon H$. This implies that we need to rescale space, time, and velocity. First of all, we rescale $z$,

$$
\xi=\frac{z}{\epsilon},-0.1 A \leq \xi \leq 1
$$

Since $t_{0}=\frac{\epsilon^{2}}{b^{2}}, b=10$, we have

$$
\eta=\frac{z}{\sqrt{t_{0}}}=\frac{z}{0.1 \epsilon}=10 \xi
$$

which means that the base state $c^{0}(\xi)=c^{0}\left(\frac{\eta}{10}\right)$ can be retrieved from the expression for $c^{0}(\eta)$, which was found in Sec. II E. When applying this scaling to boundary conditions (39), we observe that

$$
\frac{d \psi}{d \xi}=-\epsilon l \psi \text { at } \xi=1 \text {. }
$$

Since we also have $\psi(=-0.1 A)=0, l$ of order one, would lead to $\psi^{\prime}(-0.1 A)=0$ in lowest order, which implies that we would only find the trivial solution $\psi=0$. This means that we need to rescale $l$ in order to have a nontrivial solution. And this implies, since $l=\sigma+k^{2}$ that we need to rescale $\sigma$ and $k$ too.

$$
\bar{l}=l \epsilon \Rightarrow \bar{k}=\epsilon k, \bar{\sigma}=\epsilon^{2} \sigma .
$$

Remark 1: Notice the difference with the infinite domain described in the appendix. The approaches deviate from here on.

Remark 2: The growth rate $\sigma$ follows in principle from the analysis. This scaling implies that we need $\bar{\sigma}$ to be of order one for consistency. This turns out to be indeed the case.

Notice that we have to be careful when rescaling the base state,

$$
v^{0}(z, t)=-S c_{z}^{0}(z, t) \Rightarrow v^{0}(\xi, t)=\frac{-S}{\epsilon} c_{\xi}^{0}(\xi, t) .
$$

Set $V^{0}=\epsilon v^{0}=-S c_{\xi}^{0}$; a similar scaling is necessary for the perturbation of the velocity $\phi$ in order to preserve the coupling between $\phi$ and $\psi$ :

$$
\Phi=\epsilon \phi, \quad V^{0}=\epsilon v^{0} .
$$

This yields the rescaled equations

$$
\begin{gathered}
\psi_{\xi \xi}=\frac{\bar{\sigma} \psi+V_{\xi}^{0} \psi+V^{0} \psi_{\xi}+c_{\xi}^{0} \Phi+\bar{k}^{2} \psi+S c^{0} \psi}{1+S c^{0}}, \\
\Phi_{\xi \xi}=\bar{F}\left(\Phi, \Phi_{\xi}, \psi, \psi_{\xi}\right),
\end{gathered}
$$

where an explicit expression for $\bar{F}$ is given in Appendix A.

The rescaled boundary conditions (40) yield

$$
\Phi=\psi=0 \text { at } \xi=-0.1 A
$$

Furthermore we have

$$
\psi_{\xi}=-\bar{l} \psi, \Phi_{\xi}=-\bar{k} \Phi-\frac{\bar{l} \bar{\sigma}-\bar{k}^{2} \epsilon R a}{\bar{l}+\bar{k}} \psi \text { at } \xi=1 .
$$

Observe, however, that not two independent parameters $\epsilon, R a$ are present, but only the product $\epsilon R a$ occurs in the equations. Therefore, we define the parameter $q$,

$$
q=\epsilon R a \text {. }
$$




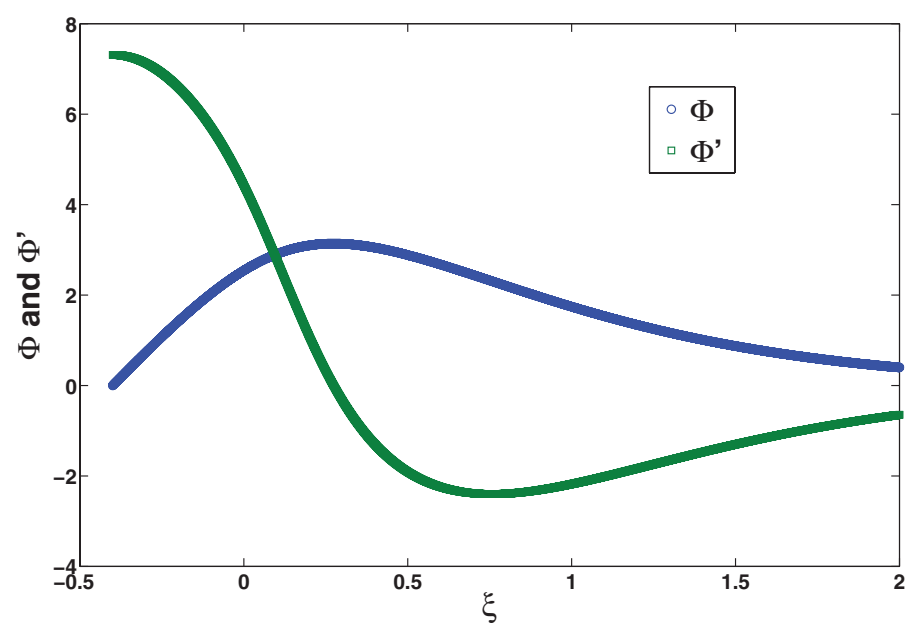

FIG. 4. The eigenfunctions $\Phi, \Phi^{\prime}$ (left) for $\bar{k}=2.0, q=20.7, A=4$.

This parameter is basically the Rayleigh number where the height of the channel is replaced by the width of the interface. In our case $R a$ is large and $\epsilon$ is small. Thus the product turns out to be of order one at the critical point, which means that $q_{c}=74.2$ in the $A=R=0$ case. This means that all terms in Eqs. (43) and (45) are of order one. In Sec. IV A, we will use different values for $A$ and $R$ and solve problem (42)-(45) numerically for different values of $q$.

\section{NUMERICAL RESULTS}

Problem (42)-(45) is solved using a shooting method. We have $\Phi(-0.1 A)=\psi(-0.1 A)=0$ according to Eq. (44). We can set $\psi^{\prime}(-0.1 A)=1$ due to normalization. Choose $\Phi^{\prime}(-0.1 A)=s_{1}$ as our first shooting parameter, which has to be determined as a part of the problem. Furthermore we set $\bar{\sigma}=s_{2}$ as our second shooting parameter; we integrate Eqs. (42) and (43) up to $\xi=1$ using a Runge-Kutta method. We use a Newton-Raphson method to find $s_{1}$ and $s_{2}$ such that Eq. (45) is satisfied. An example of the resulting eigenfunctions for $\bar{k}=2.0, q=20.7, A=4$ is given in Figs. 4 and 5. The solution for $-0.1 A<\xi<1$ is the numerical solution, the solution for $1<\xi<2$ is the analytical solution (see Eqs. (37) and (38)). The matching at $\xi=1$ yields $\Phi^{\prime}(0)$ and $\bar{\sigma}$.

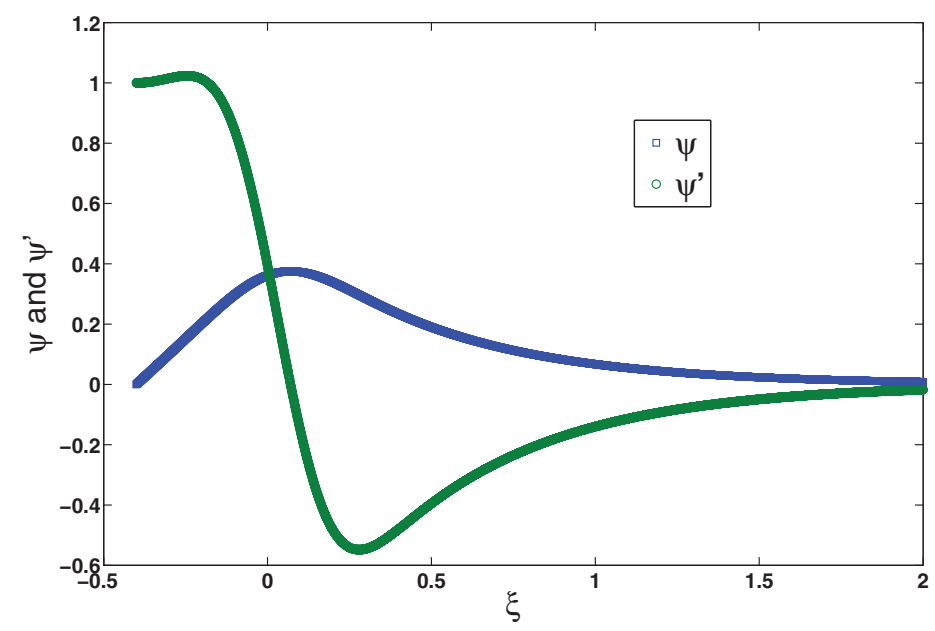

FIG. 5. The eigenfunctions $\psi, \psi^{\prime}$ (right) for $\bar{k}=2.0, q=20.7, A=4$. 


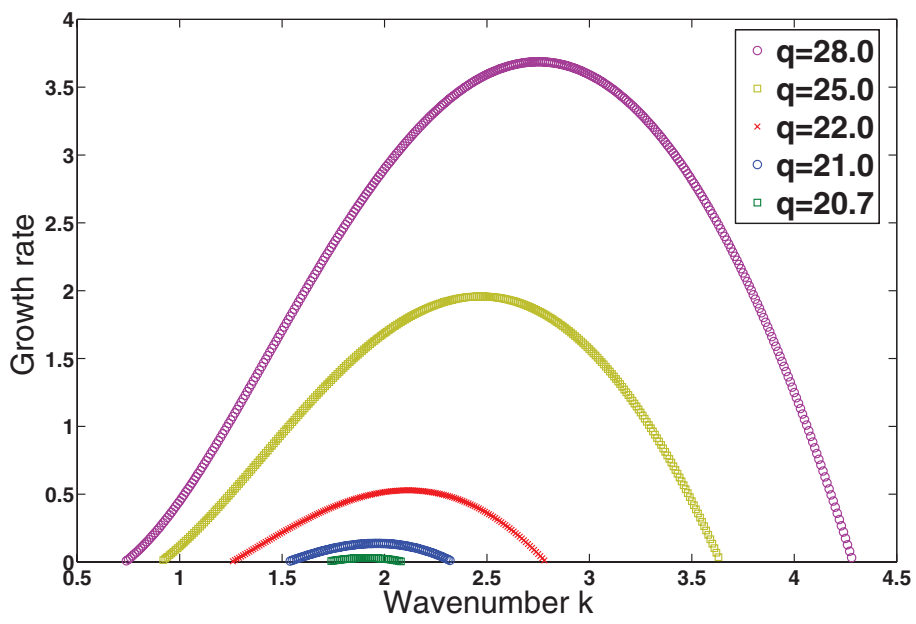

FIG. 6. Dispersion relations $\bar{\sigma}(\bar{k})$ for $A=4$.

\section{A. Results for different values of $A$ and $R$}

We only need to vary one parameter, $q$, while we keep $A$ and $R$ constant. The dispersion relations $\bar{\sigma}(\bar{k})$ for $20.7<q<28$ is given in Fig. 6 . The critical value of $q, q_{c}$ and the critical wavenumber follow from Fig. 7 for fixed $A$; the results are summarized in Table I.
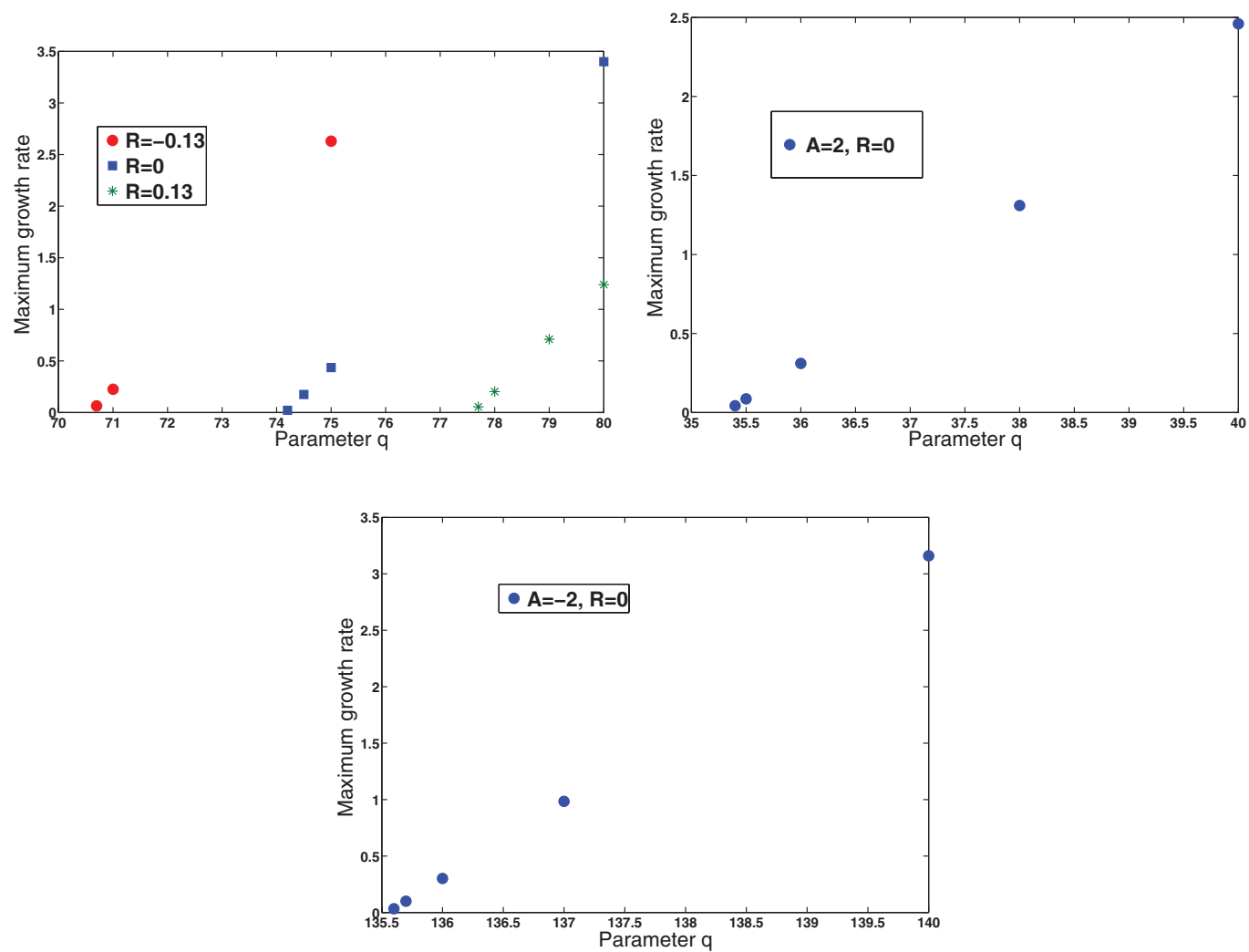

FIG. 7. Maximal values of $\bar{\sigma}$ as a function of $q$ for various choices of $A$ and $R$. 
TABLE I. Dependence of $q_{c}=\epsilon_{c} R a$ and of the critical wavenumber $k_{c}$ on the parameters $A$, where $A$ is a measure of the interface movement, i.e., $z_{\text {top }}=-A \sqrt{t}$ and $R$, where the dimensionless viscosity is $\mu=1+R c$. The error estimates are inferred from Fig. 6 as the maximum and minimal wavenumber at zero growth rate and from Fig. 7 as the critical $q$ also at zero maximum growth rate using visual inspection of the actual data. The values in the first two columns are based on typical field parameters, i.e., a reservoir at $2200 \mathrm{~m}$ depth with a temperature of $76^{\circ} \mathrm{C}$, a project time of 10 years, a interface movement of $4.7 \mathrm{~m}$, and a correlation for in situ viscosity for a $2 \mathrm{M}$ salt solution at reservoir temperature. The other columns are representative for our laboratory experiments (see Sec. V).

\begin{tabular}{lcccccccc}
\hline \hline & $\mathrm{R}=0.25$ & \multicolumn{3}{c}{$\mathrm{R}=0.13$} \\
$A$ & $q_{c}$ & $k_{c}$ & $q_{c}$ & $k_{c}$ & $q_{c}$ & $k_{c}$ & $q_{c}$ & $k_{c}$ \\
\hline-2 & & & $141.5 \pm 0.5$ & $6.7 \pm 0.2$ & $135.6 \pm 0.1$ & $6.65 \pm 0.05$ & $129.6 \pm 0.1$ & $6.65 \pm 0.1$ \\
0 & & & $77.7 \pm 0.2$ & $4.4 \pm 0.2$ & $74.2 \pm 0.1$ & $4.3 \pm 0.05$ & $70.7 \pm 0.1$ & $4.2 \pm 0.2$ \\
2 & & & $37.0 \pm 0.1$ & $2.8 \pm 0.05$ & $35.4 \pm 0.1$ & $2.8 \pm 0.2$ & $33.4 \pm 0.1$ & $2.8 \pm 0.2$ \\
4 & $20.7 \pm 0.1$ & $1.9 \pm 0.2$ & & & & & & \\
\hline \hline
\end{tabular}

\section{B. Retrieving critical time and critical wavelength}

In Sec. IV A, the parameters $q_{c}$ and $\bar{k}_{c}$ are determined for a fixed value of $R a$; in this section we will summarize how various other quantities are derived from those three parameters. From Eqs. (3) and (46), we have

$$
\begin{gathered}
t=\frac{\epsilon^{2}}{b^{2}}, b=10, \\
q=\epsilon R a,
\end{gathered}
$$

which means that we find a dimensionless critical time

$$
t_{c}=0.01 \frac{q_{c}^{2}}{R a^{2}}=\frac{a_{c}}{R a^{2}},
$$

where we defined $a_{c}=0.01 q_{c}^{2}$. This yields in dimensional form (seconds)

$$
\hat{t}_{c}=\frac{a_{c}}{R a^{2}} \frac{H^{2}}{D}=a_{c} \frac{\left(\mu_{0} \varphi\right)^{2} D}{\left(k_{0} \Delta \hat{\rho} g\right)^{2}} .
$$

According to Eq. (41) we have

$$
\bar{k}=\epsilon k,
$$

which means that we find for the dimensionless critical wavelength $\lambda_{c}$

$$
\lambda_{c}=\frac{2 \pi}{k_{c}}=\frac{2 \pi}{\epsilon_{c} \bar{k}_{c}}=\frac{2 \pi q_{c}}{\bar{k}_{c} R a}=\frac{b_{c}}{R a},
$$

where we defined $b_{c}=\frac{2 \pi q_{c}}{\bar{k}_{c}}$. This yields in dimensional form (meters)

$$
\hat{\lambda}_{c}=\frac{b_{c}}{R a} H=b_{c} \frac{\mu_{0} \varphi D}{k_{0} \Delta \hat{\rho} g} .
$$

We observe that the upward movement of the interface leads to earlier destabilization of the interface (smaller values of $q_{c}$ hence smaller critical times). We also see that although the critical wavenumber becomes smaller, also the critical wavelength becomes smaller (since $\lambda_{c}$ scales with $\left.q_{c} / k_{c}\right)$.

For $A=0$ and $R=0$, i.e., with zero interface velocity and without viscosity effects, we find

$$
q_{c}=74.2, \bar{k}_{c}=4.3 .
$$


TABLE II. Summary of physical input parameters and variables.

\begin{tabular}{lccc}
\hline \hline Physical quantity & Symbol & Value & Unit \\
\hline Dimensionless interface position / $-\sqrt{t}$ & $\mathrm{~A}$ & -2 to 4 & $(\ldots)$ \\
Concentration at interface & $c_{0}$ & 0.034 & $(\mathrm{M} / \mathrm{atm})$ \\
Initial concentration & $c_{i}$ & 0 & $(\mathrm{M} / \mathrm{atm})$ \\
$\mathrm{CO}_{2}$ concentration & $c$ & $0-0.034$ & $(\mathrm{M} / \mathrm{atm})$ \\
Diffusion coefficient & $D$ & $1.5 \times 10^{9}$ & $\left(\mathrm{~m}^{2} / \mathrm{s}\right)$ \\
Acceleration due to gravity & $g$ & 9.81 & $\left(\mathrm{~m} / \mathrm{s}^{2}\right)$ \\
Height of the reservoir & $H$ & 100 & $(\mathrm{~m})$ \\
Permeability & $k_{0}$ & In experiment: square of fracture & \\
& & width / 12, in field: $10^{-15}-10^{-11}$ & $\left(\mathrm{~m}^{2}\right)$ \\
Wave number & $k$ & Arbitrary & $(\ldots)$ \\
Length of reservoir & $L$ & Arbitrary & $(\mathrm{m})$ \\
Pressure & $P$ & 8 & $(\mathrm{bar})$ \\
Modified Rayleigh number & $q$ & Eq. $(46)$ & $(\ldots)$ \\
Rayleigh factor & $R a$ & Eq. $(17)$ & $(\ldots)$ \\
Rayleigh number & $R a$ & Eq. $(20)$ & $(\ldots)$ \\
Dimensionless time & $t$ & & $(\ldots)$ \\
Dimensionless velocities in x- and z-direction & $\bar{u}, \bar{v}$ & Result of calculations & $(\ldots)$ \\
Dimensionless coordinates & $x$, & & $(\ldots)$ \\
Width of interface & $\epsilon$ & Small & $(\ldots)$ \\
Self similar dimensionless coordinate & $\eta$ & $z / \sqrt{t}$ & $(\ldots)$ \\
Viscosity & $\mu$ & Eq. $(19)$ & $($ Pa s) \\
Fluid density & $\rho$ & Eq. $(19)$ & $\left(\mathrm{kg} / \mathrm{m}^{3}\right)$ \\
Growth rate & $\sigma$ & Arbitrary & $(\ldots)$ \\
Porosity & $\varphi$ & 1 & $(\ldots)$ \\
\hline \hline
\end{tabular}

And this yields according to Eq. (47) to a critical time

$$
t_{c}=\frac{55}{R a^{2}} .
$$

Furthermore we obtain the critical wavelength $\lambda_{c}$ according to Eq. (49):

$$
\lambda_{c}=\frac{108}{R a} \text {. }
$$

\section{The effect of the viscosity variation and the interface movement}

Figure 7 presents the critical growth rate, $\bar{\sigma}_{c}$, as a function of parameter $q$ for different values of $R$. The positive $R$ means that dissolution of $\mathrm{CO}_{2}$ in the liquid leads to increase in its viscosity, e.g., water- $\mathrm{CO}_{2}$ system. Conversely negative $R$ is indication of the decrease of the liquid viscosity with increasing $\mathrm{CO}_{2}$ concentration, which is typical for most $\mathrm{CO}_{2}$-oil systems. It is obvious from this figure that increase in the viscosity of the liquid results in more stability, i.e., the time for onset of the natural convection increases (Table II).

Furthermore, we observe from Table I that downward interface movement $(A<0)$ stabilizes the interface, whereas upward movement of the interface $(A>0)$ tends to destabilize the interface.

\section{EXPERIMENTAL RESULTS}

To validate the model, we compare our results with the experimental results of Mojaddamzadeh. ${ }^{34}$ The transparent cell consists of two parallel plates with the distance of about $8 \mathrm{~mm}$ from each other. The system remains closed during the experiment. $\mathrm{CO}_{2}$ is introduced from top to the cell containing water at a pressure of 8 bars. The Schlieren method ${ }^{35,36}$ is used to visualize the initiation and growth of the fingers. Considering the geometry of the setup, the Rayleigh number of 
this system becomes

$$
R a=\frac{\Delta \hat{\rho} g k_{0} H}{\varphi D \mu_{0}}=\frac{2 \times 9.81 \times(0.008 \times 0.008 / 12) \times 0.07}{\left(1 \times 2 \times 10^{-9} \times 0.001\right)}=3.5 \times 10^{6} .
$$

Use Eq. (49),

$$
\lambda_{c}=\frac{83}{R a_{c}}=1.6 \mu \mathrm{m} \text {. }
$$

This value is much smaller than the measured value of $0.1 \mathrm{~mm}$. Several factors can contribute to this discrepancy. First we should note that the initial stages of the fingering behavior are not detectable within the accuracy of the experimental measurement. Another reason is the questionable validity of Darcy's law for this system since the distance between two plates is large. We find a miscible Rayleigh Taylor length $L_{M R T}=58 \mu \mathrm{m}$ using the parameters from Eq. (53), which is larger than the separation of the plates $(8 \mathrm{~mm})$. In fact to improve the match one would need to solve Stokes equation. ${ }^{37}$ In the Hele-Shaw case including a Brinkman correction (see the discussion in Sec. I B 1) and the references therein) leads to an estimate of $\lambda=2.3 d=18 \mathrm{~mm}$. Solving the Brinkman problem in our case would be a useful direction of future research. Finally it appears from the figures in Ref. 34 that the length of the mixing zone is larger than $L_{M R T}$. The extension of the mixing zone might play a stabilizing role ${ }^{24}$ (which leads to larger $\lambda_{c}$ ). In this case a dispersion coefficient should replace the molecular diffusion coefficient $D$ in the calculations of the Rayleigh number.

We also compare our results to the experimental results of Foster ${ }^{38}$ who conducted experiments for salt water and found an unstable wavelength of $\lambda=1.8 \mathrm{~mm}$, which is an upper bound due to difficulties in the measurement. They reported $R a=2.63 \times 10^{3}$ for their experiment, from which we obtain $\lambda_{c}=0.6 \mathrm{~mm}$. We also recalculate the Rayleigh number based on the data reported in Foster ${ }^{38}$

$$
\begin{aligned}
& \Delta \rho=190 \mathrm{~kg} / \mathrm{m}^{3}, H=1.4 \times 10^{-2} \mathrm{~m}, L=0.13 \times 10^{-3} \mathrm{~m}, \mu=2 \times 10^{-3} \mathrm{Pas}, \\
& D=1.5 \times 10^{-9} \mathrm{~m}^{2} / \mathrm{s},
\end{aligned}
$$

which yields $R a=1.2 \times 10^{4}$ and $\lambda_{c}=0.13 \mathrm{~mm}$. Clearly, for validation of the model further experiments are required.

Furthermore we estimate the value of $A$ for experiments. ${ }^{32,39}$ After $1800 \mathrm{~s}$, a volume increase of about $2 \%$ is observed for the $\mathrm{CO}_{2}-\mathrm{C}_{10}$ system, which means that we have (in dimensionless units):

$$
t^{0}=1800 \frac{D}{H^{2}}=\frac{1800 \times 2 \times 10^{-9}}{0.2 \times 0.2}=9 \times 10^{-5} .
$$

This means that we find (in dimensionless units)

$$
A=\frac{0.02}{\sqrt{9 \times 10^{-5}}} \approx 2 .
$$

We also estimated $A$ for reservoir parameters, which yields $A=4$ (see the Introduction).

\section{A. General discussion}

The critical time for the onset of natural convection, $t_{c}$, scales with $\mathrm{Ra}^{-2}$. The critical wavelength of the fastest growing finger scales with $\mathrm{Ra}^{-1}$. The dimensional critical time and wavelength become (see Eqs. (48) and (50))

$$
\begin{gathered}
\hat{t}_{c}=a_{c} \frac{(\mu \varphi)^{2} D}{(k \Delta \rho g)^{2}} \\
\lambda_{c}=b_{c} \frac{\mu \varphi D}{k \Delta \rho g}
\end{gathered}
$$

where, for the case that the interface is fixed, the injected $\mathrm{CO}_{2}$ does not affect the brine viscosity and the capillary transition zone is neglected, the values of $a_{c}$ and $b_{c}$ were found to be 55 and 108 , respectively. The values are of the same order of magnitude as the values reported in $\mathrm{Xu}$ 
et al. ${ }^{9}$ who obtain $\left(a_{c}=75.19, b_{c}=96.23\right)$ and in Ennis-King et al.,${ }^{8}$ who obtain $\left(80<a_{c}<100\right.$, $\left.100<b_{c}<120\right)$. The discrepancy between the values are mainly due to different approaches of linear stability analysis. Furthermore, we take (small) density variations entering the zeroth order state into account, which are neglected in Xu et al. ${ }^{9}$ and Ennis-King et $a l .{ }^{8}$ Our analysis already signals a single unstable mode, which means that we obtain lower a lower value for the critical time and hence for $a_{c}$.

We extended the stability analysis by assuming a moving interface between the $\mathrm{CO}_{2}$-brine mixture and the brine. To obtain analytical solutions we assumed that the interface moves proportional to square-root of time. Our results indicate that neglecting interface movement can lead to errors in estimation of the amount of stored $\mathrm{CO}_{2}$ due to solubility. To clarify this, we assume an aquifer with a permeability of $100 \mathrm{mD}$ and porosity of $20 \%$ that is saturated with brine with a viscosity of $0.5 \mathrm{mPa}$ s. With the assumption that the dissolution of $\mathrm{CO}_{2}$ increases the brine density by $10 \mathrm{~kg} / \mathrm{m}^{3}$, and taking $D=5 \times 10^{-9} \mathrm{~m}^{2} / \mathrm{s}$ without considering interface movement the critical time is calculated to be about 331 days. If an upward velocity of the interface is considered this time decreases to about 26 days. If the permeability of the aquifer (or in general the Rayleigh number) decreases the difference becomes even more significant. For example choosing an aquifer permeability of $20 \mathrm{mD}$ and keeping the other parameters constant, with and without considering the interface movement, the critical time becomes 8280 and 644 days, respectively. Therefore, in this example, neglecting the interface movement can lead to more than 21 years of difference and therefore the amount of dissolved $\mathrm{CO}_{2}$ can be over-estimated. If the interface moves downwards the convection initiates later than the case for which interface movement is not considered.

\section{CONCLUSIONS}

The influence of viscosity and interface velocity on the stability of a diffusive boundary layer is investigated using a QSSA. Our analysis is of relevance for $\mathrm{CO}_{2}$ sequestration and solvent based enhanced oil recovery projects. In the case of $\mathrm{CO}_{2}$-brine system dissolution of $\mathrm{CO}_{2}$ leads to an increase of the viscosity of brine, while in most $\mathrm{CO}_{2}$-oil systems dissolution of $\mathrm{CO}_{2}$ results in lower liquid viscosity. Moreover the dissolution of $\mathrm{CO}_{2}$ can lead to volume increase (swelling) or volume decrease (shrinkage) of $\mathrm{CO}_{2}$ liquid systems, i.e., the location of the interface cannot be assumed fixed.

The initial stage of the instability is investigated, where the width of the layer scales $\sim \epsilon$ and the time $\sim \epsilon^{2}$. Rescaling the corresponding equations leads to a cascade of rescalings. The relevant bifurcation parameter is found to be $q=\epsilon R a$, which is basically the Rayleigh number with the width of the interface as the characteristic length. This implies that a convective instability sets in once the width of the diffusive boundary layer exceeds a certain value.

Furthermore it is clear that the bifurcation parameter $q$ implies the well knowing scalings of the critical time $\sim \frac{1}{R a^{2}}$ and the critical wavelength $\sim \frac{1}{R a}$. The simulations are used to estimate both proportionality constants.

The analysis also shows the existence of two timescales: the base state evolves on a slower timescale than the perturbations, which formally justifies the QSSA. Notice that this separation of scales is not present when the boundary conditions are imposed at $\pm \infty^{13}$ which means that the QSSA in that case is questionable.

We observe that the presence of the upstream interface movement enhances the instability. In our analysis we have assumed that the interface position is proportional to $\sqrt{t}$. Furthermore we see that the enhanced viscosity of the liquid due to $\mathrm{CO}_{2}$ dissolution stabilizes the interface.

\section{APPENDIX A: EXPRESSION FOR $\boldsymbol{F}$ AND $\overline{\boldsymbol{F}}$}

$$
F=\frac{A \phi+B \phi_{z}+C \psi+D \psi_{z}}{\left(1+R c^{0}\right)\left(1+S c^{0}\right)^{2}}
$$

where

$$
A=-S c_{z z}^{0}+S^{2}\left(c_{z}^{0}\right)^{2}-S^{2} c_{z z}^{0} c^{0}-R c^{0} S v^{0} c_{z}^{0}-S v^{0} c_{z}^{0}+k^{2}+2 k^{2} S c^{0}+k^{2} S^{2}\left(c^{0}\right)^{2}
$$




$$
\begin{gathered}
+k^{2} R c^{0}-R S\left(c_{z}^{0}\right)^{2} \\
+2 k^{2} R S\left(c^{0}\right)^{2}+k^{2} R S^{2}\left(c^{0}\right)^{3}-R S c^{0} c_{z z}^{0}-R S^{2}\left(c^{0}\right)^{2} c_{z z}^{0} \\
B=-c_{z}^{0}\left(R+S+S^{2} c^{0}+3 R S c^{0}+2 R S^{2}\left(c^{0}\right)^{2}\right) \\
C=-S v_{z z}^{0}-2 R a k^{2} S c^{0}-S^{2} v_{z z}^{0} c^{0}+S^{2} \sigma c_{z}^{0}+S^{2} c_{z}^{0} v_{z}^{0}-R S \sigma c^{0} v^{0}-R S c^{0} v^{0} v_{z}^{0}-S \sigma v^{0} \\
-S^{2} k^{2} v^{0} c^{0}-S v^{0} v_{z}^{0} \\
-S k^{2} v^{0}-R a k^{2}+R k^{2} v^{0}+R S k^{2} v^{0} c^{0}-R S \sigma c_{z}^{0}-R S c_{z}^{0} v_{z}^{0}-R S c^{0} v_{z z}^{0}-R S^{2}\left(c^{0}\right)^{2} v_{z z}^{0}-R a k^{2} S^{2}\left(c^{0}\right)^{2} \\
D=-S\left(\sigma+\left(v^{0}\right)^{2}+2 v_{z}^{0}+S \sigma c^{0}+2 S c^{0} v_{z}^{0}-S v^{0} c^{0} c_{z}^{0}+R c^{0}\left(v^{0}\right)^{2}+R c_{z}^{0} v^{0}+R \sigma c^{0}+R S \sigma\left(c^{0}\right)^{2}\right. \\
\left.+2 R c^{0} v_{z}^{0}+2 R S v_{z}^{0}\left(c^{0}\right)^{2}\right) \\
\bar{F}=\frac{\bar{A} \Phi+\bar{B} \phi_{\xi}+\bar{C} \psi+\bar{D} \psi_{\xi}}{\left(1+R c^{0}\right)\left(1+S c^{0}\right)^{2}}
\end{gathered}
$$

where

$$
\begin{gathered}
\bar{A}=-S c_{\xi \xi}^{0}+S^{2}\left(c_{\xi}^{0}\right)^{2}-S^{2} c_{\xi \xi}^{0} c^{0}-R c^{0} S V^{0} c_{\xi}^{0}-S V^{0} c_{\xi}^{0}+\bar{k}^{2}+2 \bar{k}^{2} S c^{0}+\bar{k}^{2} S^{2}\left(c^{0}\right)^{2}+\bar{k}^{2} R c^{0}-R S\left(c_{\xi}^{0}\right)^{2} \\
\bar{B}=-c_{\xi}^{0}\left(R+S+S^{2} c^{0}+3 R S c^{0}+2 R S^{2}\left(c^{0}\right)^{2}\right) \\
\bar{C}=-S V_{\xi \xi}^{0}-2 R a \epsilon \bar{k}^{2} S c^{0}-S^{2} V_{\xi \xi}^{0} c^{0}+S^{2} \bar{\sigma} c_{\xi}^{0}+S^{2} c_{\xi}^{0} V_{\xi}^{0}-R S \bar{\sigma} c^{0} V^{0}-R S c^{0} V^{0} V_{\xi}^{0}-S \bar{\sigma} V^{0}-S^{2} \bar{k}^{2} V^{0} c^{0} \\
-S V^{0} V_{\xi}^{0}-S \bar{k}^{2} V^{0}-R a \epsilon \bar{k}^{2}+R \bar{k}^{2} V^{0}+R S \bar{k}^{2} V^{0} c^{0}-R S \bar{\sigma} c_{\xi}^{0}-R S c_{\xi}^{0} V_{\xi}^{0}-R S c^{0} V_{\xi \xi}^{0}-R S^{2}\left(c^{0}\right)^{2} V_{\xi \xi}^{0} \\
-R a \epsilon \bar{k}^{2} S^{2}\left(c^{0}\right)^{2} \\
\bar{D}=-S\left(\bar{\sigma}+\left(V^{0}\right)^{2}+2 V_{\xi}^{0}+S \bar{\sigma} c^{0}+2 S c^{0} V_{\xi}^{0}-S V^{0} c^{0} c_{\xi}^{0}+R c^{0}\left(V^{0}\right)^{2}+R c_{\xi}^{0} V^{0}+R \bar{\sigma} c^{0}+R S \bar{\sigma}\left(c^{0}\right)^{2}\right. \\
\left.+2 R c^{0} V_{\xi}^{0}+2 R S V_{\xi}^{0}\left(c^{0}\right)^{2}\right)
\end{gathered}
$$

\section{APPENDIX B: DERIVATION OF THE ANALYTICAL EXPRESSION FOR THE DISPERSION RELATION IN AN INFINITE DOMAIN}

In this appendix we derive the equation for the dispersion relation $\sigma(k)$ given in, e.g., Tan and Homsy, ${ }^{12}$ Eq. (42), and Rogerson and Meiburg, ${ }^{13}$ Eqs. (87)- (89) with $\theta=\frac{\pi}{2}$. We are not aware of the existence of a detailed derivation of those equations. Since the derivation is nontrivial, it seems worthwhile to add the derivation here. 
An infinite domain is considered in this case and an initial concentration jumping from zero to one at $\tilde{z}=0$. The base state is given as

$$
c^{0}(\tilde{z})=\frac{1}{\sqrt{4 \pi t}} \int_{-\infty}^{\tilde{z}} e^{-s^{2} / 4 t} d s, \frac{\partial c^{0}}{\partial \tilde{z}}=\frac{1}{\sqrt{4 \pi t}} e^{-\tilde{z}^{2} / 4 t}, \bar{v}=\bar{v}^{0} .
$$

The equations for first order perturbation are

$$
\begin{gathered}
\left(\frac{d^{2}}{d \tilde{z}^{2}}+R c_{\tilde{z}}^{0} \frac{d}{d \tilde{z}}-k^{2}\right) \phi=k^{2}\left(\bar{v}^{0} R-\overline{R a} S e^{(S-R) c^{0}}\right) \psi, \\
\left(\frac{d^{2}}{d \tilde{z}^{2}}-k^{2}-\sigma\right) \psi=c_{\tilde{z}}^{0} \phi
\end{gathered}
$$

which are the same as Eqs. (52) and (53) from Rogerson and Meiburg, ${ }^{13}$ with $\theta=\frac{\pi}{2}$ and a different choice of non-dimensionalization, which is in terms of the velocity of the waves $(G=\overline{R a}$ and $\left.\bar{v}^{0}=1\right)$ in that paper.

\section{Analysis in two regions}

An analytical expression for the dispersion relation can only be found in the limit $t_{0} \rightarrow 0$. We set

$$
t_{0}=\frac{\epsilon^{2}}{4 a}
$$

we fix $\epsilon>0$ and small and we take $a$ to infinity. This choice for $t_{0}$ yields

$$
\frac{\partial c_{0}}{\partial \tilde{z}}=\frac{1}{\epsilon} \sqrt{\frac{a}{\pi}} e^{-a \frac{\tilde{z}^{2}}{\epsilon^{2}}}
$$

which is negligible for large values of $|\tilde{z}|$. This means that we can study the problem in two regimes: large $|\tilde{z}|$ in Appendix B 1 a and small $|\tilde{z}|$ Appendix B 1 b.

\section{a. Analytic solutions for large $|\tilde{z}|$}

In this case, Eqs. (33)-(34) reduce to

$$
\left(\frac{d^{2}}{d \tilde{z}^{2}}-k^{2}\right) \phi=k^{2}\left(\bar{v}_{0} R-\overline{R a} S\right) \psi,\left(\frac{d^{2}}{d \tilde{z}^{2}}-l^{2}\right) \psi=0
$$

for $\tilde{z}>0$ and

$$
\left(\frac{d^{2}}{d \tilde{z}^{2}}-k^{2}\right) \phi=k^{2}\left(\bar{v}_{0} R-\overline{R a} S e^{S-R}\right) \psi,\left(\frac{d^{2}}{d \tilde{z}^{2}}-l^{2}\right) \psi=0
$$

for $\tilde{z}<0$. The solutions are

$$
\psi=c_{5} e^{l \tilde{z}}, \phi=c_{6} e^{k \tilde{z}}+c_{5} \frac{k^{2} G_{0}}{l^{2}-k^{2}} e^{l \tilde{z}} \text { for } \tilde{z}<0,
$$

where $G_{0}=\bar{v}^{0} R-\overline{R a} S$ and

$$
\psi=c_{7} e^{-l \tilde{z}}, \phi=c_{8} e^{-k \tilde{z}}+c_{7} \frac{k^{2} G_{1}}{l^{2}-k^{2}} e^{-l \tilde{z}} \text { for } \tilde{z}>0,
$$

where $G_{1}=\bar{v}^{0} R-\overline{R a} S e^{S-R}$. We have at both $\tilde{z}=-\epsilon$ and $\tilde{z}=\epsilon$ four matching conditions: four are used to eliminate $c_{5}-c_{8}$ and we are left with two conditions at $\tilde{z}=-\epsilon$ and $\tilde{z}=\epsilon$ :

$$
\psi^{\prime}=l \psi, \phi^{\prime}=k \phi+\frac{k^{2} G_{0}}{l+k} \psi \text { at } \tilde{z}=-\epsilon
$$


and

$$
\psi^{\prime}=-l \psi, \phi^{\prime}=-k \phi-\frac{k^{2} G_{1}}{l+k} \psi \text { at } \tilde{z}=\epsilon
$$

\section{b. Small values of $|\tilde{z}|$ : A power series solution reveals the structure}

We have to solve Eqs. (B1) and (B2) for small values of $|\tilde{z}|$. This means that we can try to find a power series solution of the form

$$
\phi(\tilde{z})=c_{1}+c_{2} \tilde{z}+d_{2} \tilde{z}^{2}+\ldots, \psi(\tilde{z})=c_{3}+c_{4} \tilde{z}+e_{2} \tilde{z}^{2}+\ldots
$$

where the coefficients $d_{2}, d_{3}, \ldots$ and $e_{2}, e_{3}, \ldots$ are determined by the ODE's in terms of $c_{1}-c_{4}$, which may be chosen freely. Solving the equations for $d_{2} . d_{3}$. . explicitly, we observe however that $d_{2} \sim \epsilon^{-1}, d_{3} \sim \epsilon^{-2}$, etc., the same for $e_{2}, e_{3}$, which means that we have

$$
\phi(x)=c_{1}+\epsilon\left(c_{2} \frac{\tilde{z}}{\epsilon}+d_{2} \frac{\tilde{z}^{2}}{\epsilon^{2}}+\ldots\right)=c_{1}+\epsilon h(\xi) .
$$

We can exploit this structure setting

$$
\begin{gathered}
\xi=\frac{\tilde{z}}{\epsilon}, \frac{d c_{0}}{d \tilde{z}}=\frac{1}{\epsilon} g(\xi), g(\xi)=\sqrt{\frac{a}{\pi}} e^{-a \xi^{2}} . \\
\phi(\xi)=c_{1}+\epsilon h(\xi), \psi(\xi)=c_{3}+\epsilon j(\xi)
\end{gathered}
$$

Substitute these expressions in Eqs. (B2) and (B3) and evaluate up to first order in $\epsilon$ :

$$
h^{\prime \prime}+R g(\xi) h^{\prime}=0
$$

$$
j^{\prime \prime}=c_{1} g(\xi)
$$

where the prime denotes differentiation with respect to $\xi$. The matching conditions have to be evaluated at $\xi= \pm 1$ and yield (up to lowest order in $\epsilon$ )

$$
j^{\prime}(-1)=l c_{3}, j^{\prime}(1)=-l c_{3}
$$

and

$$
h^{\prime}(-1)=k c_{1}+\frac{k^{2} G_{0}}{l+k} c_{3}, h^{\prime}(1)=-k c_{1}-\frac{k^{2} G_{1}}{l+k} c_{3}
$$

(because $\frac{d \phi}{d \tilde{z}}=\frac{1}{\epsilon} \frac{d \phi}{d \xi}=\frac{1}{\epsilon} \epsilon h^{\prime}=h^{\prime}$ )

Integration of Eq. (B5) yields

$$
j^{\prime}(\xi)=j^{\prime}(0)+c_{1} \int_{0}^{\xi} g(\xi) d \xi \Rightarrow j^{\prime}(1)=j^{\prime}(0)+\frac{1}{2} c_{1}, j^{\prime}(-1)=j^{\prime}(0)-\frac{1}{2} c_{1},
$$

because

$$
\lim _{a \rightarrow \infty} \sqrt{\frac{a}{\pi}} \int_{0}^{1} e^{-a \xi^{2}} d \xi=\lim _{a \rightarrow \infty} \sqrt{\frac{1}{\pi}} \int_{0}^{\sqrt{a}} e^{-u^{2}} d u=\frac{1}{2}
$$

Combining this with Eq. (B6) yields

$$
c_{1}=-2 l c_{3}, \text { and } j^{\prime}(0)=0 .
$$

Separate and integrate Eq. (B4) to obtain

$$
\frac{h^{\prime \prime}}{h^{\prime}}=-R g(\xi) \Rightarrow \ln \left(h^{\prime}(\xi)\right)=-R \int_{0}^{\xi} g(\xi) d \xi+\ln \left(h^{\prime}(0)\right),
$$


which yields

$$
h^{\prime}(-1)=h^{\prime}(0) e^{R / 2}, h^{\prime}(1)=h^{\prime}(0) e^{-R / 2} .
$$

Use the boundary conditions (B7) to find

$$
\left(-2 l k+\frac{k^{2} G_{0}}{l+k}\right) e^{-R / 2}=\left(2 l k-\frac{k^{2} G_{1}}{l+k}\right) e^{R / 2} .
$$

Solving for $l$ we find

$$
2 l k(l+k)-k^{2} \tilde{R}=0
$$

where

$$
\tilde{R}=\frac{G_{1} e^{R}+G_{0}}{1+e^{R}}=\bar{v}^{0} R-\overline{\operatorname{Ra}} S \frac{1+e^{S}}{1+e^{R}}
$$

which leads to

$$
l=\frac{1}{2}\left(-k+\sqrt{k^{2}+2 k \tilde{R}}\right) .
$$

Finally using $\sigma=l^{2}-k^{2}$ we obtain

$$
\sigma=\frac{k}{2}\left(\tilde{R}-k-\sqrt{k^{2}+2 k \tilde{R}}\right),
$$

which is Eq. (89) in Rogerson and Meiburg. ${ }^{13}$

${ }^{1}$ L. Gmelin, Gmelins Handbuch der Anorganischen Chemie, System-Nr. 14: 8. Auflage. Kohlenstoff, Teil C3, Verbindungen., 8th ed. (Springer, Berlin, 1973).

${ }^{2}$ E. Lindeberg and D. Wessel-Berg, "Vertical convection in an aquifer column under a gas cap of $\mathrm{CO}_{2}$," Energy Convers. Manage. 38, S229-S234 (1997).

${ }^{3}$ R. Farajzadeh, P. Ranganathan, P. Zitha, and J. Bruining, "The effect of heterogeneity on the character of density driven natural convection of $\mathrm{CO}_{2}$ overlying a brine layer," Adv. Water Resour. 34, 327-339 (2011).

${ }^{4}$ R. Farajzadeh, B. Meulenbroek, D. Daniel, A. Riaz, and J. Bruining, "An empirical theory for gravitationally unstable flow in porous media," Comput. Geosci. 17, 515-527 (2013).

${ }^{5}$ R. Farajzadeh, H. Salimi, P. L. J. Zitha, and H. Bruining, "Numerical simulation of density-driven natural convection in porous media with application for $\mathrm{CO}_{2}$ injection projects," Int. J. Heat Mass Transfer 50, 5054-5064 (2007).

${ }^{6} \mathrm{Ch}$. Yang and $\mathrm{Y}$. Gu, "Accelerated mass transfer of $\mathrm{CO}_{2}$ in reservoir brine due to density-driven natural convection at high pressures and elevated temperatures," Ind. Eng. Chem. Res. 45, 2430-2436 (2006).

${ }^{7}$ A. Riaz, M. Hesse, H. A. Tchelepi, and F. M. Orr, "Onset of convection in a gravitationally unstable diffusive boundary layer in porous media," J. Fluid Mech. 548, 87-111 (2006).

${ }^{8}$ J. Ennis-King, I. Preston, and L. Paterson, "Onset of convection in anisotropic porous media subject to a rapid change in boundary conditions," Phys. Fluids 17, 084107 (2005).

${ }^{9} \mathrm{X}$. Xu, S. Chen, and D. Zhang, "Convective stability analysis of the long-term storage of carbon dioxide in deep saline aquifiers," Adv. Water Resour. 29, 397-407 (2006).

${ }^{10}$ K. B. Haugen and A. Firoozabadi, "Composition at the interface between multi-component nonequilibrium fluid phases," J. Chem. Phys. 130, 064707 (2009).

${ }^{11}$ R. Farajzadeh, P. L. J. Zitha, and J. Bruining, "Enhanced mass transfer of $\mathrm{CO}_{2}$ into water: Experiment and modeling," Ind. Chem. Res. 48, 6423-6431 (2009).

${ }^{12}$ C. T. Tan and G. M. Homsy, "Stability of miscible displacements in porous media: Rectilinear flow," Phys. Fluids 29, 3549-3556 (1986).

${ }^{13}$ A. Rogerson and E. Meiburg, "Shear stabilization of miscible displacement processes in porous media," Phys. Fluids A 5, 1344-1355 (1993).

${ }^{14}$ S. Rapaka, S. Chen, R. J. Pawar, P. H. Stauffer, and D. Zhang, "Non-modal growth of perturbations in density-driven convection in porous media," J. Fluid Mech. 609, 285-303 (2008).

${ }^{15} \mathrm{~J}-\mathrm{P}$ Caltagirone, "Stability of a saturated porous layer subject to a sudden rise in surface temperature: Comparison between the linear and energy methods," Q. J. Mech. Appl. Math. 33, 47-58 (1980).

${ }^{16}$ D. A. S. Rees, A. Selim, and J. P. Ennis-King, "The instability of unsteady boundary layers in porous media," Emerging Topics in Heat and Mass Transfer in Porous Media (Springer Netherlands, 2008), pp. 85-110.

${ }^{17}$ J. W. Gardner and J. G. J. Ypma, "An investigation of phase-behavior macroscopic-bypassing interaction in CO2 flooding," SPEJ 24, 508-520 (1984).

${ }^{18}$ H. C. Brinkman, "A calculation of the viscous force exerted by a flowing fluid on a dense swarm of particles," Appl. Sci. Res. 1, 27-34 (1949).

${ }^{19}$ J. Zeng, Y. C. Yortsos, and D. Salin, "On the brinkman correction in unidirectional hele-shaw flows," Phys. Fluids 15, 3829-3836 (2003). 
${ }^{20}$ J. Fernandez, P. Kurowski, L. Limat, and P. Petitjeans, "Wavelength selection of fingering instability inside hele-shaw cells," Phys. Fluids 13, 3120-3125 (2001).

${ }^{21}$ J. Fernandez, P. Kurowski, P. Petitjeans, and E. Meiburg, "Density-driven unstable flows of miscible fluids in a hele-shaw cell," J. Fluid Mech. 451, 239-260 (2002).

${ }^{22}$ Y. C. Yortsos and M. Zeybek, "Dispersion driven instability in miscible displacement in porous media," Phys. Fluids 31, 3511-3518 (1988).

${ }^{23}$ J. C. Bacri, N. Rakotomalala, D. Salin, and R. Woumeni, "Miscible viscous fingering: Experiments versus continuum approach," Phys. Fluids A 4, 1611-1619 (1992).

${ }^{24}$ J. Martin, N. Rakotomalala, and D. Salin, "Gravitational instability of miscible fluid in a hele-shaw cell," Phys. Fluids 14, 902-905 (2002).

${ }^{25}$ J. W. Elder, "The unstable thermal interface," J. Fluid Mech. 32, 69-96 (1968).

${ }^{26}$ T. D. Foster, "Stability of a homegeneous fluid cooled uniformly from above," Phys. Fluids 8, 1249-1257 (1965).

${ }^{27}$ P. M. Gresho and R. L. Sani, "The stability of a fluid layer subjected to a step change in temperature: transient vs. frozen time analyses," Int. J. Heat Mass Transfer 14, 207-221 (1971).

${ }^{28}$ M. C. Kim, K. Y. Kim, and S. Kim, "The onset of transient convection in fluid-saturated porous layer heated uniformly from below," Int. Commun. Heat Mass Transfer 31, 53-62 (2004).

${ }^{29}$ M. C. Kim, "Onset of buoyancy driven convection in isotropic porous media heated from below," Korean J. Chem. Eng. 27, 741-747 (2010).

${ }^{30}$ G. I. Barenblatt, V. M. Entov, and V. M. Ryzhyk, Theory of Fluid Flows Through Natural Rocks (Springer, 1990).

${ }^{31}$ V. S. Mitlin, Nonlinear Dynamics of Reservoir Mixtures (CRC PressINC, 1993).

${ }^{32}$ R. A. Farajzadeh, H. Delil, P. L. J. Zitha, and H. Bruining, "Enhanced mass transfer of $\mathrm{CO}_{2}$ into water and oil by natural convection," in Proceedings of Europec/EAGE Conference and Exhibition, 11-14 June 2007, London, UK (Society of Petroleum Engineers, 2007).

${ }^{33}$ S. Bando, F. Takemura, M. Nishio, E. Hihara, and M. Akai, "Viscosity of aqueous $\mathrm{NaCl}$ solutions with dissolved $\mathrm{CO}_{2}$ at (30 to 60) degrees C and (10 to 20) MPa," J. Chem. Eng. Data 49, 1328-1332 (2004).

${ }^{34}$ A. Mojaddamzadeh, " $\mathrm{CO}_{2}$ sequestration: Visualization of natural convection flow of $\mathrm{CO}_{2}$ in water by Schlieren method," Master's thesis, Delft University of Technology, The Netherlands, 2009.

${ }^{35}$ R. Khosrokhavar, G. Elsinga, A. Mojaddamzadeh, R. Farajzadeh, and J. Bruining, "Visualization of natural convection flow of (sub) and (super) critical $\mathrm{CO}_{2}$ in aqueous and oleic systems by applying Schlieren method," in Proceeding of Society of Petroleum Engineers SPE EUROPEC/EAGE Annual Conference and Exhibition held in Vienna, Austria, 23-26 May 2011 (Society of Petroleum Engineers, 2011).

${ }^{36}$ G. S. Settles, Schlieren and Shadowgraph Techniques: Visualizing Phenomena in Transperent Media (Springer-Verlag, 2001).

${ }^{37} \mathrm{C}-\mathrm{Y}$. Chen and E. Meiburg, "Miscible porous media displacements in the quarter five-spot configuration. Part 2. Effect of heterogeneities," J. Fluid Mech. 371, 269-299 (1998).

${ }^{38}$ L. L. Green and T. D. Foster, "Secondary convection in a hele shaw cell," J. Fluid Mech. 71, 675-691 (1975).

${ }^{39}$ R. Farajzadeh, A. Barati, H. A. Delil, J. Bruining, and P. L. J. Zitha, "Mass transfer of $\mathrm{CO}_{2}$ into water and surfactant solutions," Pet. Sci. Technol. 25, 1493-1511 (2007). 